\title{
Vinylic Addition Polynorbornene in Catalysis
}

Rodrigo García-Loma ${ }^{[a]}$ and Ana C. Albéniz ${ }^{*[a]}$

Dedicated to Prof. Pablo Espinet on the occasion of his $70^{\text {th }}$ birthday

\section{VA-PNB}

$\left(\mathrm{CH}_{2}\right)_{n} \mathrm{SnBu}_{2} \mathrm{R}$

$\sim\left(\mathrm{CH}_{2}\right)_{n} \mathrm{SnHBu}_{2}$

\section{VA-PNB}

Supported Reagents

Organocatalysts

VA-PNB $N$

VA-PNB

$\sim\left(\mathrm{CH}_{2}\right)_{4}$
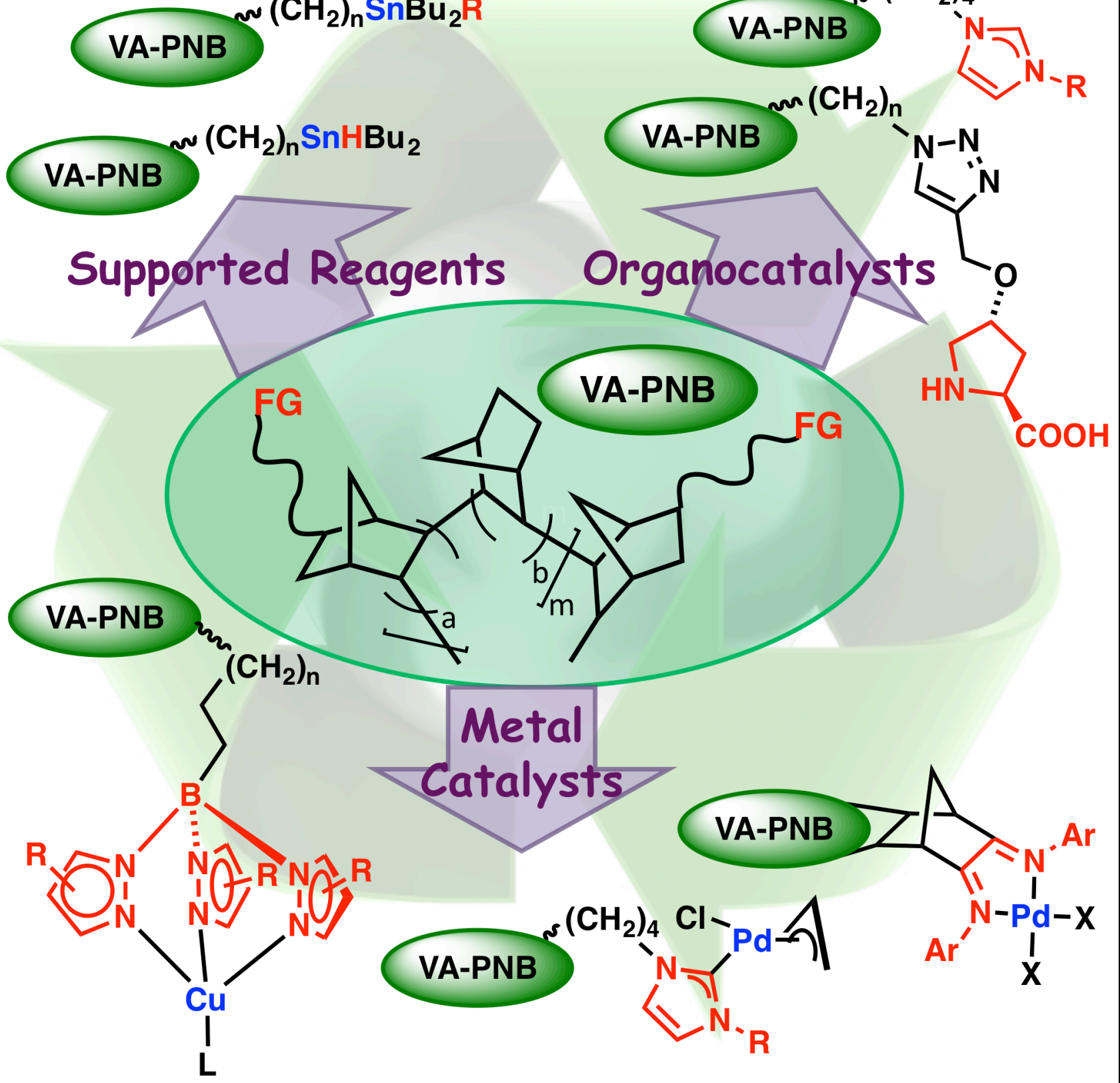
Abstract: Vinylic addition polynorbornes (VA-PNB) result from the insertion polymerization of norbornene or specific norbornene derivatives catalyzed by transition metal complexes. The VA-PNB skeleton is completely aliphatic and keeps the bicyclic structure of norbornene. This saturated polymer is thermally and chemically very stable and it is a very robust scaffold to support catalysts or reagents for catalytic applications. Several VA-PNBs are now available with suitable functional groups (halogen, alkenyl, carbonates) that can be used as starting materials to introduce the functionalization of choice by post-polymerization reactions. This has been applied to anchor organocatalysts on VA-PNB as well as ligands that can be used to synthesize supported metal catalysts. The reported examples of the use of VA-PNB-linked catalysts and their recyclability will be presented. VA-PNBs have also proved useful in the context of organotin chemistry to solve the problem of tin contamination by an efficient separation and the reuse of tin-byproducts. Its uses in the Stille cross coupling reaction and tin-hydride radical processes will be discussed.

\section{Introduction}

The use of polymers in organic synthesis and catalysis is widespread because they bring practical advantages and contribute to the sustainability of the synthetic protocols. ${ }^{[1]}$ Polymers are commonly employed as supports of a reaction partner, either the catalyst or a reagent, which are anchored through strong covalent bonds. A strong linkage is important to preserve the polymer-bound reactive moiety throughout the desired transformation, and this moiety can be introduced either by controlled polymerization of suitable functionalized monomers or by post-polymerization functionalization techniques. The solubility of polymers is often quite different to that of discrete small molecules and therefore, their separation should be simple. The insoluble polymeric reagents are easily recovered by filtration; the polymers that are soluble in the reaction conditions, which is an attractive property to increase reactivity, can be later precipitated by changing the solvent or the temperature, and then filtered from the reaction mixture. This easy separation results in the recovery and potential reuse of catalysts or, for supported reagents, in easier purification of the target products from the polymer-bound byproducts. The use of polymeric materials as supports in catalysis has been thoroughly reviewed over the years and interesting accounts on soluble, ${ }^{[2]}$ insoluble polymeric supports, ${ }^{[3]}$ or both, ${ }^{[4]}$ as well as other applications, ${ }^{[5]}$ can be found in the literature.

Most polymeric supports involved in catalytic reactions are polystyrene resins (PS), polyethylene glycol derivatives (PEG) or copolymers that mix both backbones (Figure 1). They have become very useful and popular because of the presence of pendant functional groups (halogen, hydroxyl, etc.) that can be transformed in a variety of ways, and because they are commercially available. Although PS and PEG are suitable for many applications, their polymer backbones include sensitive positions (aromatic rings, benzylic positions, C-heteroatom bonds), which could undergo undesired reactions depending on the reaction conditions. For example, the aromatic backbone of the polystyrene could interfere in electrophilic aromatic substitutions such as Friedel-Crafts acylation and nitration. ${ }^{[6]}$ Also, the benzylic positions in PS are susceptible of undergoing an attack by radicals if used in radical processes. Less reactive polymeric scaffolds that increase the range of processes where they can be used could offer practical advantages.

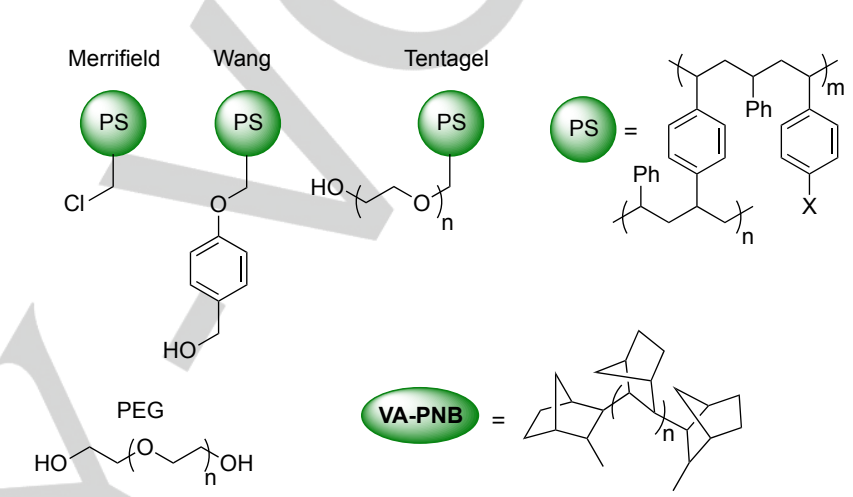

Figure 1. Some common commercially available resins and vinylic addition polynorbornene (VA-PNB).

A saturated all-aliphatic polymer backbone would not undergo interferences during most reactions, being close to an ideal spectator solid support and leaving the supported functionality as the only possible reactive center. Also, having a chemically robust skeleton would increase the chances of getting reusable materials, which is an important and desirable feature for any supported reagent. However, there are just a few examples of all-aliphatic polymers based on polyethylene, ${ }^{[}{ }^{7} \quad$ ] polyisobutylene ${ }^{[8]}$ or polypropylene skeletons, ${ }^{[9]}$ which have been used as supports of organocatalysts, ${ }^{[7]}$ or transition metal catalysts such as $\mathrm{Pd},{ }^{[8 a, 9]}$ or Ru complexes. ${ }^{[8 \mathrm{~b}]}$ Vinylic addition polynorbornenes (VA-PNBs) are also saturated materials that meet all the requirements for a robust spectator scaffold (Figure 1). We have been working for some time on the synthesis and application of VA-PNBs and they have proved to be interesting and useful supports. This review collects the examples to date of the use of these polynorbornene materials in catalysis.

[a] Dr. R. García-Loma and Prof. Dr. A. C. Albéniz IU CINQUIMA/Química Inorgánica Universidad de Valladolid

47011 Valladolid, Spain

E-mail: albeniz@qi.uva.es 


\section{Vinylic addition polynorbornene (VA-PNB) vs other polynorbornenes.}

Norbornene (bicyclo[2.2.1]hept-2-ene) is a strained olefin which easily undergoes polymerization in different ways, depending on the catalyst and reaction conditions used. ${ }^{[10]}$ Scheme 1 shows the two more common polymerization mechanisms and the main differences in the resulting materials. Vinylic addition polymerization (Scheme 1, a) leads to an aliphatic skeleton that preserves the bicyclic structure of norbornene (VA-PNB). This polymer is obtained by an insertionpolymerization mechanism using a suitable metal complex as catalyst and examples are known for both early and late transition metals. ${ }^{[11,12]}$ The polymers are chemically and thermally stable, with high glass transition temperatures and optical transparency. The introduction of the functionality of choice in this saturated polymer backbone could be more challenging since the insertion-polymerization of norbornene is not tolerant of many functional groups, so the direct polymerization of substituted monomers generally leads to polymers in very low yields and, when a copolymerization with norbornene is attempted, low incorporation of the functional monomers. However, as it will be discussed below, this can be circumvented by the polymerization of selected norbornene derivatives and post-polymerization functionalization of the resulting materials.

The polymerization of norbornene by a cationic mechanism using powerful electrophiles also leads to an all-alliphatic polymer backbone. However, the molecular weight of the polymers are generally low which makes them less suitable to be used as solid supports.

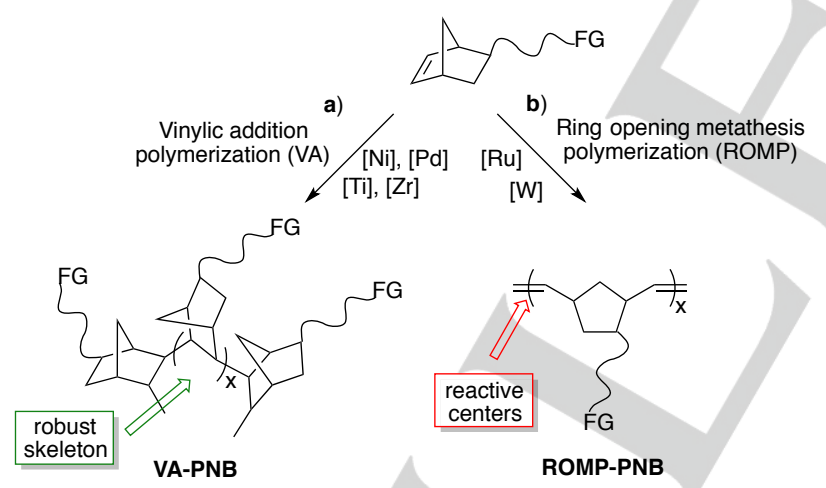

Scheme 1. Main polymerization mechanisms of norbornene and resulting materials $(F G=$ functional group)

The ring opening metathesis polymerization of norbornene is very effective by Grubbs' type ruthenium catalysts or by tungsten derivatives leading to ROMP-PNB (Scheme 1, b). This polymerization is highly tolerant to the presence of functional groups on norbornene and allows to synthesize many functionalized polynorbornenes, ${ }^{[13]}$ some of them used as supports in catalysis. [14 ] However, ROMP-PNBs have a polyunsaturated backbone that make this type of skeleton highly sensitive to the catalytic conditions used and they can even play a role of a reagent competing with the actual substrates in the catalytic reaction. This has been shown for ROMP-PNBsupported tungsten complexes: when these catalysts are used in cross-metathesis reactions of alkenes, the polymer backbone also reacts leading to oligomeric soluble norbornene byproducts. ${ }^{[15]}$ We have also demonstrated that the disubstituted olefins of ROMP-PNB do insert in Pd-aryl bonds, becoming a potential reactive center in palladium-catalyzed cross coupling reactions. The reaction of a ROMP-PNB with the complex $\left[\operatorname{PdBr}\left(\mathrm{C}_{6} \mathrm{~F}_{5}\right)(\mathrm{NCMe})_{2}\right]$ leads to the incorporation of the pentafluorophenyl group in the polymer backbone as clearly shown by ${ }^{19} \mathrm{~F}$ NMR (Scheme 2). ${ }^{[16]}$
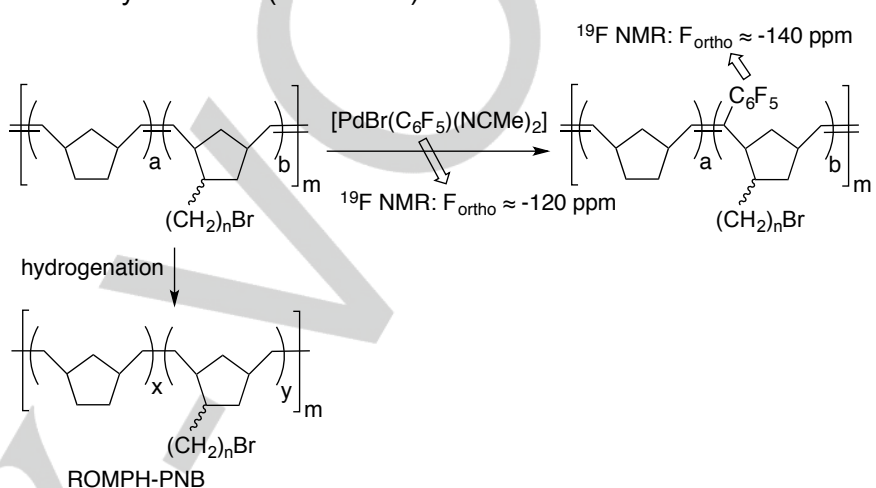

Scheme 2. Reactivity of a ROMP-PNB backbone and hydrogenation to a saturated ROMPH-PNB.

ROMP-PNBs can be converted into saturated scaffolds by hydrogenation of the double bonds in the main chain. Although

Rodrigo García-Loma received his BSc degree in Chemistry from the University of Valladolid in 2010. He just obtained his Ph. D. at the University of Valladolid under the supervision of Prof. Ana C. Albéniz. His work has dealt with the development of allalliphatic polymers derived from norbornene and their use in tin-radical processes and gold catalysis

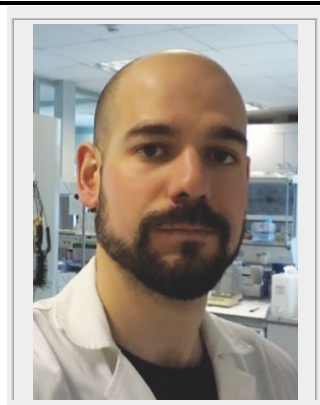

Ana C. Albéniz is a Full Professor of Inorganic Chemistry at the University of Valladolid. She obtained her Ph. D. at the University of Valladolid and worked as a postdoctoral Fulbright fellow in Prof. Crabtree's group at Yale University. In 1991 she returned to Valladolid as an Associate Professor. Since 2007 she is a Full Professor working in the Centre for Innovation in Chemistry and Advanced Materials (CINQUIMA) at the University of Valladolid. Her research interests include the

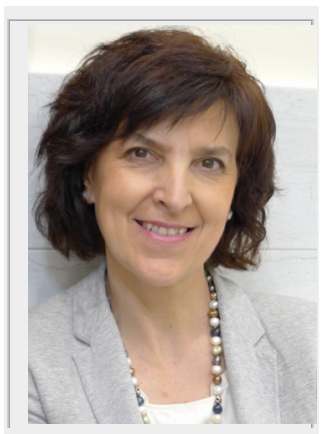
development and mechanistic study of new metal-catalyzed reactions applied to organic synthesis, the synthesis of new polymers, and their application in catalysis as recyclable supports for catalyst and reagents. 
the hydrogenation processes are not compatible with every functional group in the polymer, ROMP-hydrogenated materials (ROMPH-PNBs) bearing groups amenable to postpolymerization functionalization have been prepared (Scheme 2). ${ }^{[16]}$ Therefore, the synthesis of ROMPH-PNBs does not benefit from the advantage of the wide scope of the direct ROMP polymerization of substituted norbornenes, but affords a saturated and chemically robust scaffold. This has been shown for a stannylated ROMPH-PNB, which has been used as support of the tin reagent in the palladium catalyzed Stille coupling. ${ }^{[16]}$ This work showed that this type of scaffold behaves in a similar way to the vinylic addition polynorbornenes which, being prepared in a lower number of steps, is a more convenient choice for catalytic applications.

\section{Functionalized vinylic addition polynorbornenes useful as starting materials.}

The direct polymerization of many substituted norbornenes by an insertion mechanism to obtain vinylic addition polynorbornenes is not an efficient reaction. Usually, those metal complexes that are very active in the polymerization of norbornene, do not work for substituted norbornenes, specially those with polar groups, or give polymer materials in low yields. When a mixture of norbornene and the substituted norbornene is copolymerized, the different reactivity of both monomers often gives polymers with a low incorporation of the substituted norbornene. Some progress has been done in the area and better catalysts for the polymerization of functionalized norbornenes have been synthesized. However, the tolerance of functional groups in this polymerization reaction is still low. A recent review by Bermeshev and Chapala gives a comprehensive account of the advances made in the field. ${ }^{[17]}$

Sometime, we took a different approach to the problem. Instead of a direct polymerization, a more convenient way would be to have a suitable starting VA-PNB that could be transformed by a wide range of conventional reactions to give the VA-PNB with the functionalization of interest. This is a post-polymerization functionalization approach, close to the strategy used by most chemists when using commercially available polystyrene resins like those depicted in Figure 1.

We succeeded in synthesizing the VA-PNBs with pendant $\omega$ bromo-alkyl chains collected in Scheme 3. The use of the nickel catalyst $\left[\mathrm{Ni}\left(\mathrm{C}_{6} \mathrm{~F}_{5}\right)_{2}\left(\mathrm{SbPh}_{3}\right)_{2}\right]$, also very active for the polymerization of norbornene, allowed us to obtain homopolymers of bromoalkyl norbornenes and also copolymers with norbornene of different composition and size. As expected, the reactivity of norbornene is higher but the polymer composition can be roughly controlled by the ratio of monomers in the feed. In this way a range of VA-PNBs with $\omega$-bromoalkyl chains of different lengths and different functionalization content could be prepared $(\mathrm{a} / \mathrm{b}=0-2.8) .{ }^{[18]}$ These haloalkyl monomers have rarely been polymerized by an insertion mechanism using other catalysts, ${ }^{[19]}$ and the above-mentioned nickel perfluoroaryl complex is the best catalyst to obtain haloalkyl VA-PNBs to date.

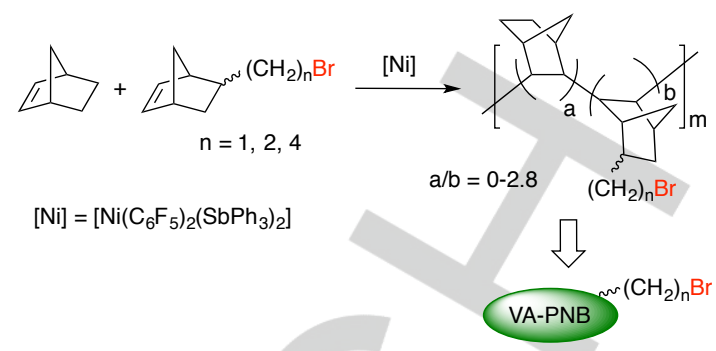

Scheme 3. Synthesis of VA-PNBs with pendant bromoalkyl groups.

The $\omega$-bromoalkyl VA-PNBs can be used as starting materials to replace the bromo group for another functionality using a range of nucleophilic substitutions, $\mathrm{HBr}$ elimination and even $\mathrm{C}-\mathrm{C}$ coupling reactions (Scheme 4). ${ }^{[18,20]}$ The synthesis of the VAPNBs bearing azido groups is an interesting transformation since this opens the possibility of using azido-alkyne cycloaddition reactions, a very useful click reaction, to anchor a variety of groups. VA-PNB- $\left(\mathrm{CH}_{2}\right)_{n} \mathrm{Br}$ materials are in a way analogues to the polystyrene resins with halobenzyl groups because of their versatility as starting materials.

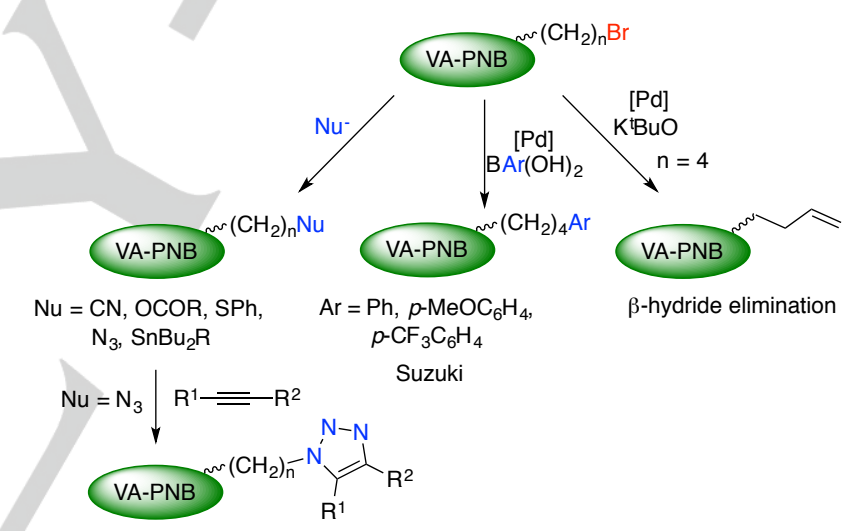

Scheme 4. Functionalization routes for VA-PNBs with bromoalkyl chains.

VA-PNBs with bromoaryl groups have also been synthesized (Scheme 5). Such functionality allows the use of palladiumcatalyzed cross coupling reactions to introduce aryl, vinyl, amino or enolate groups in the polymer (Scheme 6). As shown in Eq. 1, the ability of these polymers to act as reagents in such reactions was demonstrated by the synthesis of a polymeric palladium derivative by oxidative addition of VA-PNB- $\mathrm{C}_{6} \mathrm{H}_{4} \mathrm{Br}$ to a $\mathrm{Pd}(0)$ derivative, forming the $\mathrm{Pd}$-aryl reaction intermediate. In this case the VA-PNB is the subject of the catalysis, being involved as the reactant and the product.

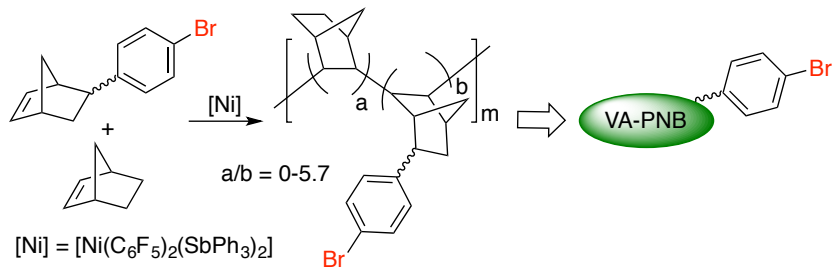

Scheme 5. Synthesis of VA-PNBs with bromoaryl substitutents 


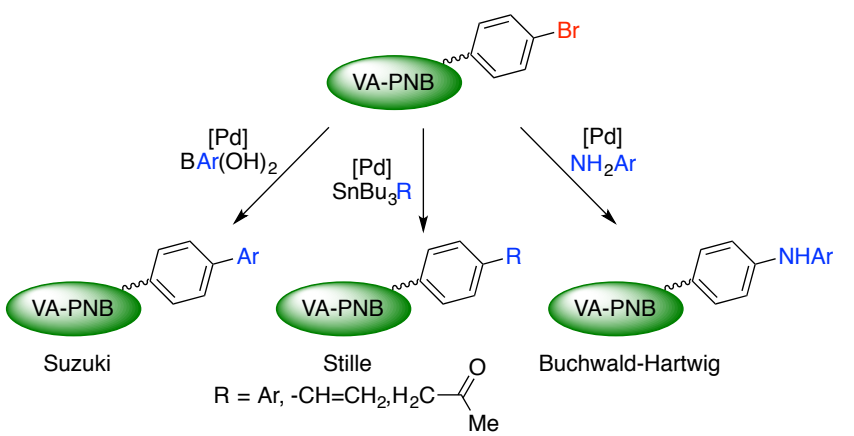

Scheme 6. Bromoaryl-substituted VA.PNBs in Pd-catalyzed cross-couplings

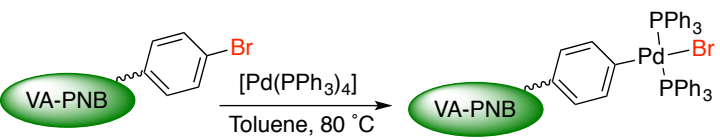

Vinylic addition polynorbornenes with pendant alkenyl groups are also useful starting materials for further functionalization. The endocyclic double bond of alkenyl norbornenes is much more reactive than the exocyclic one due to strain, and the polymerization of this type of monomers can be brought about by a variety of metal catalysts to give VA-PNBs with the exocyclic unsaturation untouched. ${ }^{[17]}$ Some alkenyl norbornene monomers are commercialy available and several reports can be found in the literature that show the polymerization and subsequent transformation of the pendant double bond in an epoxide, ${ }^{[21]}$ or an alcohol. ${ }^{[1 \mathrm{a}, \mathrm{b}]}\left[\mathrm{Ni}\left(\mathrm{C}_{6} \mathrm{~F}_{5}\right)_{2}\left(\mathrm{SbPh}_{3}\right)_{2}\right]$ has also shown to be a suitable catalyst for the synthesis of alkenyl VAPNBs of different chain lengths (Scheme 7). ${ }^{[22]}$
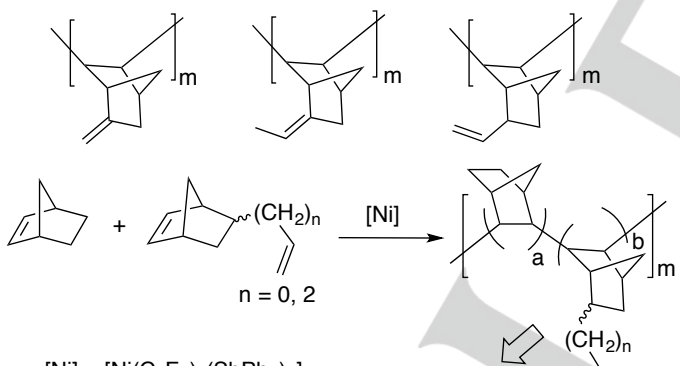

$[\mathrm{Ni}]=\left[\mathrm{Ni}\left(\mathrm{C}_{6} \mathrm{~F}_{5}\right)_{2}\left(\mathrm{SbPh}_{3}\right)_{2}\right]$

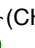

VA-PNB

Scheme 7. Examples of VA-PNBs with pendant alkenyl groups and copolymers NB/alkenyINB.

VA-PNBs in Schemes 3-7 are obtained as powdery solids and their solubility depends on the molecular weight and the composition of the copolymers. Usually, the higher the amount of norbornene (lower functionalization) the higher the molecular weight of the copolymer and therefore the solubility decreases. All VA-PNBs are insoluble in hydrocarbons and polar solvents such as DMSO, DMF, alcohols and water. On the other hand, it has been shown that freshly synthesized VA-PNBs are conformationally metastable and the thermal treatment in the solid state ${ }^{[23]}$ or in solvents like DMF induces a change in properties including a decrease in solubility. ${ }^{[24]}$ This has been attributed to the rotation of the norbornene bicyles of the rigid structure leading to the thermodynamically stable form. ${ }^{[23]}$ Thus, by this process it is easy to generate insoluble VA-PNBs that can be used to immobilize reagents or catalysts. In contrast to most polystyrene resins, VA-PNBs experience less swelling when soaked. In the cases when swelling has been observed the volume of the polymer increases by a factor of 1.5-2, but for other VA-PNBs this volume increase is not significant. This is not a drawback for reactivity, as it will be shown below.

\section{Vinylic addition polynorbornenes as support of organocatalysts}

The field of organocatalysis may greatly benefit from the use of solid supports, including robust polymers such as VA-PNBs. The organic molecules that catalyze a given transformation are often expensive or difficult to prepare, especially for enantioselective reactions. Therefore, the recovery of the catalyst at the end of the reaction is important and a solid support allows it, as well as an efficient and simple separation of the catalyst from the products. This may save tedious purification procedures and avoid the generation of extra waste. Vinylic addition polynorbornenes have been used to anchor organocatalysts of two different types: $\mathrm{N}$-heterocyclic carbenes and proline derivatives.

The first example of a VA-PNB supported organocatalyst is the polymeric $\mathrm{N}$-heterocyclic carbene shown in Scheme $8{ }^{[25]}$ The nucleophilic substitution of an imidazol for bromide in VA-PNB$\left(\mathrm{CH}_{2}\right)_{4} \mathrm{Br}(1)$ leads to supported imidazolium salts (2) that can be subsequently deprotonated to give the corresponding $\mathrm{N}$ heterocyclic carbenes (NHCs). This method allows the use of polymer 1 as starting material of a variety of NHCs with different substituents on one of the nitrogens, by a straightforward nucleophilic substitution reaction just choosing the suitable imidazole. It is a modular approach that allows catalyst diversity in contrast with other polymeric NHCs used in organocatalysis, which rely on the polymerization of monomers containing the preformed NHC precursor, generally the imidazolium salt, so the polymer needs to be synthesized from scratch for every substituent. ${ }^{[26]}$

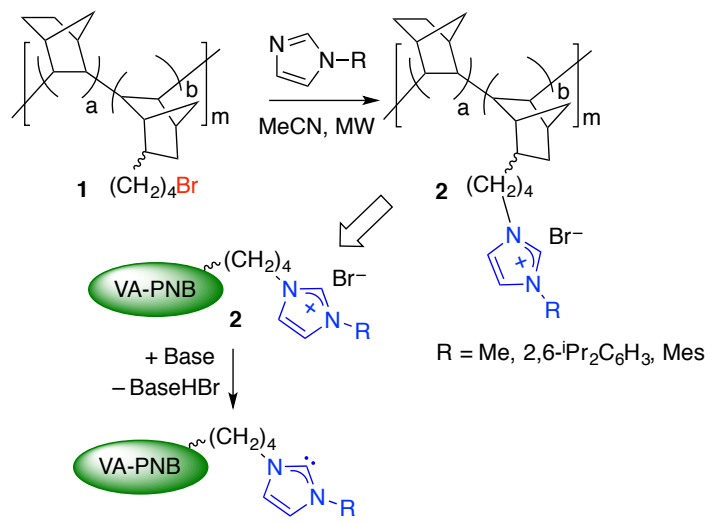

Scheme 8. Synthesis of VA-PNB supported N-heterocyclic carbenes. 
Two different reactions were tested with the VA-PNB-supported imidazolium salt $2(\mathrm{R}=\mathrm{Mes})$ : the conversion of $\alpha, \beta$-unsaturated aldehydes into saturated esters and the synthesis of $\gamma$ butyrolactones (Scheme 9). In both cases DBU was used as base to deprotonate the imidazolium salt 2 and generate the free carbene. A simple filtration allows to separate the polymer from the products after each reaction, and this polymer can be reused at least five times with no loss of activity. After repeated reuses, the polymeric $\mathrm{NHC}$ was protonated with $\mathrm{HBF}_{4}$ and the resulting imidazolium 2 (tetrafluoroborate salt) was stored in the air at room temperature. After several days the recovered polymer 2 can be used again in the reaction showing the same activity as in the first use (Scheme 9, a, 6th cycle). The catalysis is heterogeneous and it occurs on the supported NHC. This was checked by carrying out a hot filtration test: the reaction of cinnamaldehyde and benzylic alcohol (Scheme 9, a) was run to a $30 \%$ conversion and the polymer was filtered; the filtrate was kept in the reaction conditions for $12 \mathrm{~h}$ but no increase in conversion occurred.

a)<smiles>[R]C=CC(=O)[CH+]c1ccccc1</smiles>

$\mathrm{OH}$

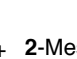
$+\underset{(10 \mathrm{~mol} \%)}{2-\mathrm{Mes})} \underset{\begin{array}{c}2 \mathrm{PhOH} \\ \text { Toluene, } \\ 48 \mathrm{~h}, 110{ }^{\circ} \mathrm{C}\end{array}}{\longrightarrow} \stackrel{\text { Filtration }}{\longrightarrow}$ $\mathrm{R}=\mathrm{Ph}, \mathrm{n}-\mathrm{Pr}$ $R^{\prime}$
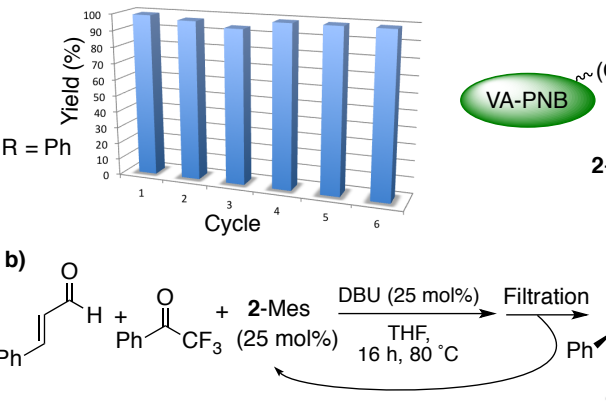

$\sim\left(\mathrm{CH}_{2}\right)_{4}$ 2-Mes

b)
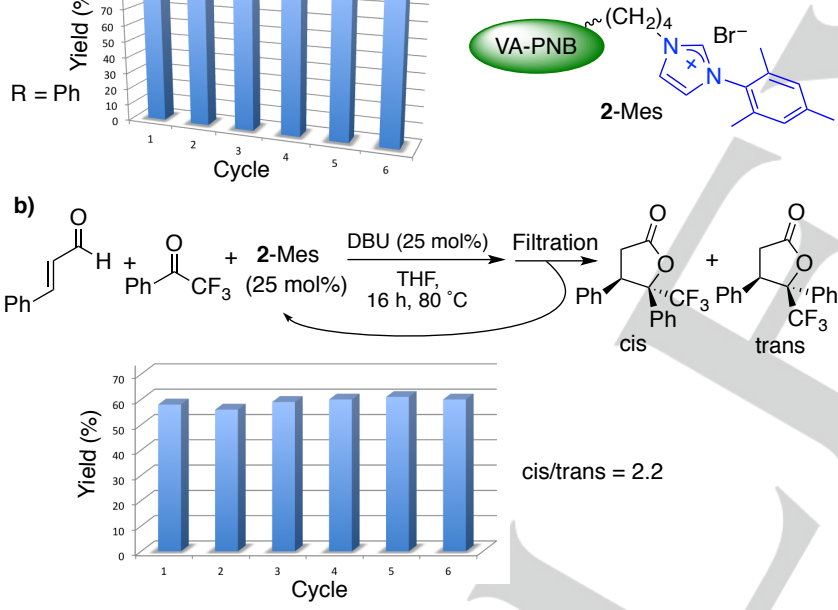

Scheme 9. VA-PNB supported N-heterocyclic carbenes as recyclable organocatalysts.

VA-PNB supported prolines have proved useful in the asymmetric aldol reaction in aqueous media. The all-aliphatic polymer backbone could mimic the hydrophobic pocket of the natural enzymes that catalyze this type of reactions, whereas the functional group anchored to the polymer bears the actual catalyst in a more hydrophilic environment. This strategy has worked well for other polymeric supports and has also been successful for VA-PNBs. L-Proline derivatives have been anchored to VA-PNBs using the Cu-catalyzed azido alkyne cycloaddition reaction (CuAAC), a click transformation that allows the attachment of the functional group to the polymer through a triazole linkage. As shown in Scheme 10, the functionalization can be achieved using a VA-PNB bearing an

azido group (3) or a VA-PNB with an alkyne moiety introduced by an ester spacer using polymer 1 as starting material (4). Both proline functionalized VA-PNBs $\mathbf{5}$ and $\mathbf{6}$ are excellent catalysts for the enantioselective reaction of a variety of aldehydes and ketones with excellent diastereomeric ratios and enantiomeric excess (Scheme 11). The supported proline derivatives can be recycled without loss of activity or selectivity, as shown in Scheme 11 for the aldol reaction between $p$-nitrobenzaldehyde and cyclohexanone. ${ }^{[27]}$

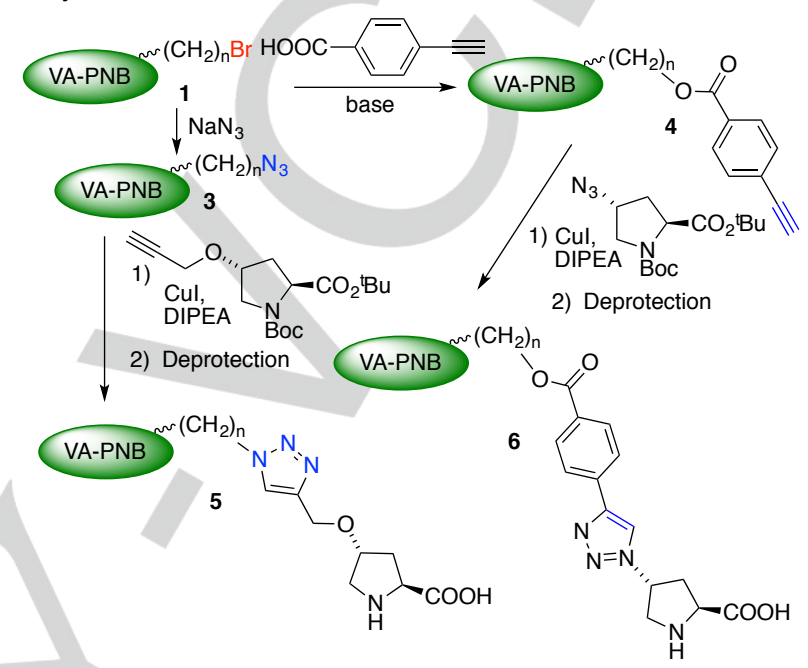

Scheme 10. Synthesis of proline derivatives supported on VA-PNB.

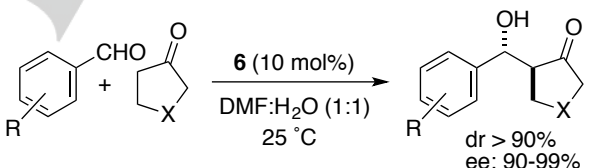

$\mathrm{R}=\mathrm{NO}_{2}, \mathrm{X}=\mathrm{CH}_{2}-\mathrm{CH}_{2}$

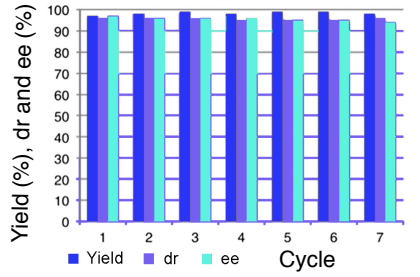

Scheme 11. Asymmetric aldol reaction catalyzed by VA-PNB-proline 6.

\section{Vinylic addition polynorbornenes as support of metal catalysts}

Supported metal catalysis is a very attractive approach that uses well-defined metal complexes, which brings all the advantages of homogeneous catalysis (single site catalytic centers, selectivity), while allowing a good separation and recovery of the catalyst, as it is possible for most heterogeneous catalysts. VAPNBs have proved to be robust supports in several processes and the functionalized polymeric materials described above as well as other precursors, allow to anchor several types of ligands to the polymeric scaffold that can be used to coordinate the metal. 
The polymeric imidazolium salts 2 are the precursors of welldefined palladium carbene complexes as shown in Eq. $2^{\left[{ }^{28]}\right.}$ Complexes 7 were characterized by solid state MAS-NMR and show the characteristic $\mathrm{Pd}-\mathrm{C}_{\text {carbene }}{ }^{13} \mathrm{C}$ NMR signal at $180 \mathrm{ppm}$. The polymeric complex 7 catalyze the Suzuki and the Negishi couplings with good results and the catalyst can be recycled.

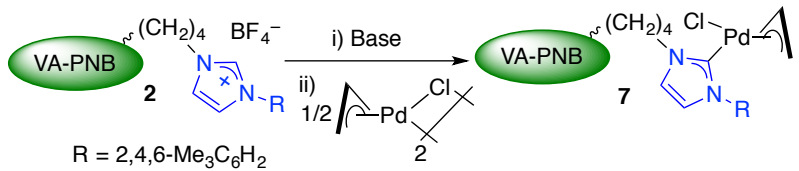

The system was studied in detail since, as it often occurs in many reactions catalyzed by supported palladium complexes, the solid material may be just the catalyst precursor and not the actual active catalyst. A Suzuki coupling was monitorized by in situ IR and the reaction showed an induction period which increased in every recycling experiment along with the total reaction time needed for complete conversion (Table 1). The SEM microscopy images showed the formation of palladium aggregates (average $65 \mathrm{~nm}$ ) on the polymer. A very small amount of palladium leaching was observed, as detected by ICP-MS of the reaction products, and this is enough to carry out the reaction in a homogeneous way as shown by a hot filtration test which reveals that, after separation of the polymer, the reaction is catalyzed by the filtrate.

Table 1. Reuse of VA-PNB-supported complex 7 in the Suzuki reaction.

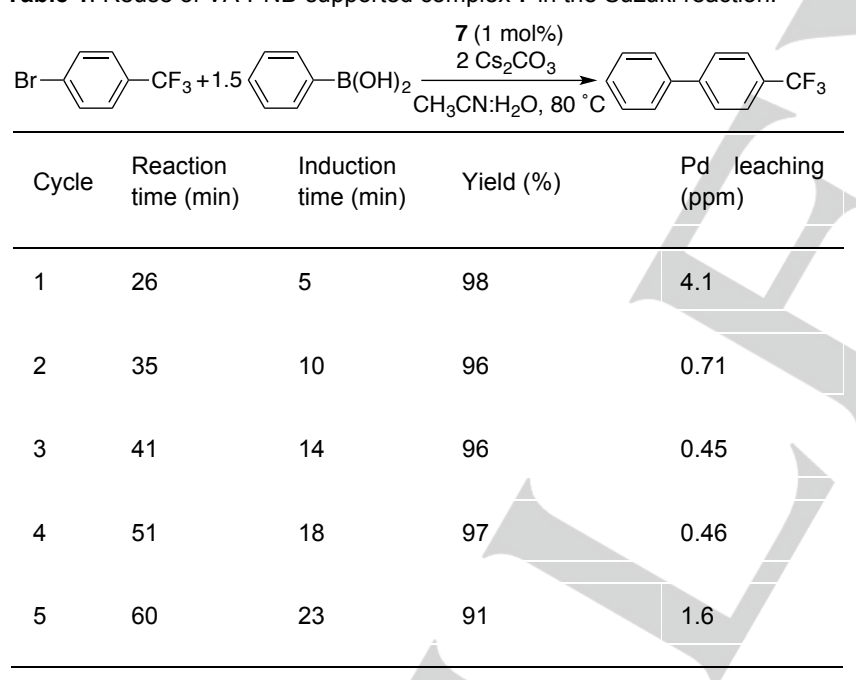

Therefore, the VA-PNB functionalized with N-heterocyclic carbenes is capable of acting as a reservoir of minute amounts of soluble palladium species (about $1 \mathrm{ppm}$ of $\mathrm{Pd}$ ) that carry out the Suzuki coupling. It occurs in a controlled way, so the polymer can be recycled many times at the expense of increasing the reaction time. This is a common scenario in cross-coupling reactions catalyzed by supported palladium complexes. Even if the reaction does not occur on the supported species, the soluble species are so active that the released amount of metal is very small, making the products little contaminated and the separation and purification easier.

Taking advantage of the bicyclic structure of norbornene, a chelating diimine ligand was built in the VA-PNB backbone as shown in Scheme 12. ${ }^{[29]}$ In order to do that, a new functionalized VA-PNB was synthesized by copolymerization of norbornene and a norbornene carbonate. The low reactivity of the latter requires the slow addition of the more reactive norbornene to the polymerization mixture; in this way enough incorporation of the functionalized monomer was achieved (Eq. 3).

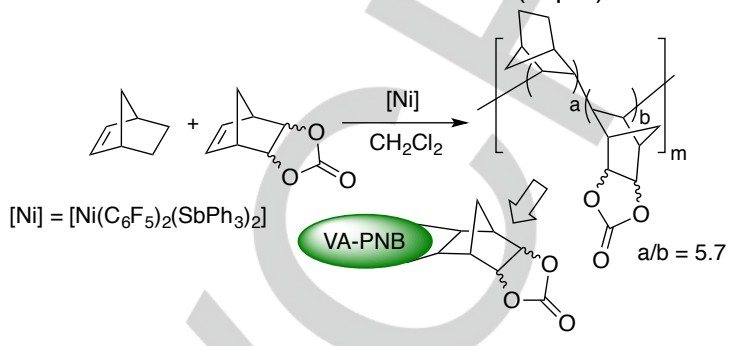

$\alpha$-Diimine ligands have been profusely used in palladium chemistry and they are very important in the context of Pdcatalyzed olefin polymerization reactions and have also found application in cross coupling reactions. A supported complex [Pd(VA-PNB-diimine)CIMe] was tested in the polymerization of ethylene but its activity was only moderate. On the other hand, complex 8 (Scheme 12) proved to be useful in Suzuki couplings and it is recyclable (Table 2). In the same way as the supported Pd-carbene complex 7, the VA-PNB diimine derivative 8 also works as a catalysts precursor of ppm amounts of soluble palladium active species. However, the deactivation of the catalyst reservoir in this case is very small and, after a slight increase in the reaction time in the first three cycles, the supported precatalyst is completely recyclable (Table 2 ).

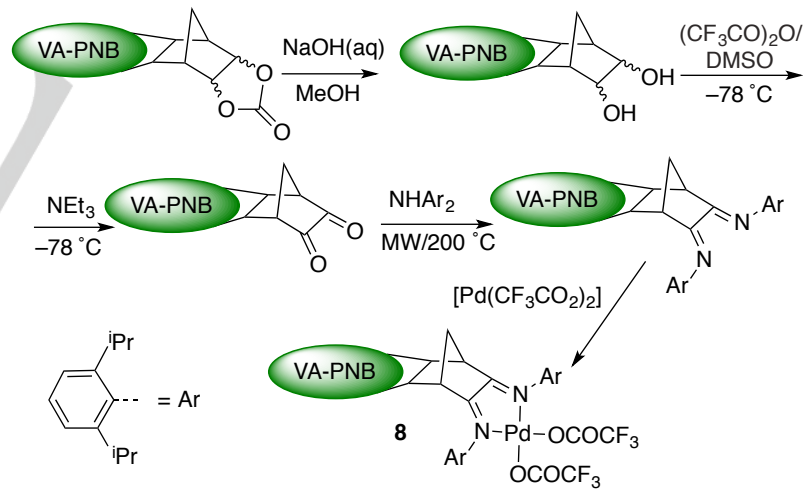

Scheme 12. Synthesis of VA-PNB-diimine ligands and supported palladium complex 8.

Table 2. Reuse of VA-PNB-supported complex 8 in the Suzuki reaction.

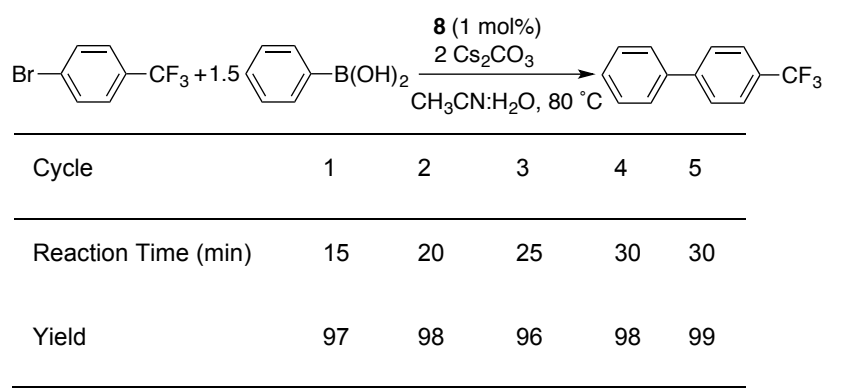


A different type of ligand that has been rarely anchored to a solid support is trispyrazolylborate (Tp). The use of some of the alkenyl substituted VA-PNBs shown in Scheme 7 as starting materials, allows a synthetic route that leads to the VA-PNBsupported trispyrazolylborate derivatives 9 with a range of substitution patterns (Scheme 13). ${ }^{[22]}$ Despite the importance of $\mathrm{Tp}$ ligands in catalysis with different metals, the use of solidsupported trispyrazolylborate complexes as recyclable catalysts had not been achieved. VA-PNB proved to be a suitable support for this purpose and the copper complexes synthesized using VA-PNB-Tps as ligands (10, Scheme 13) were active in several carbene and nitrene transfer reactions (Scheme 14).

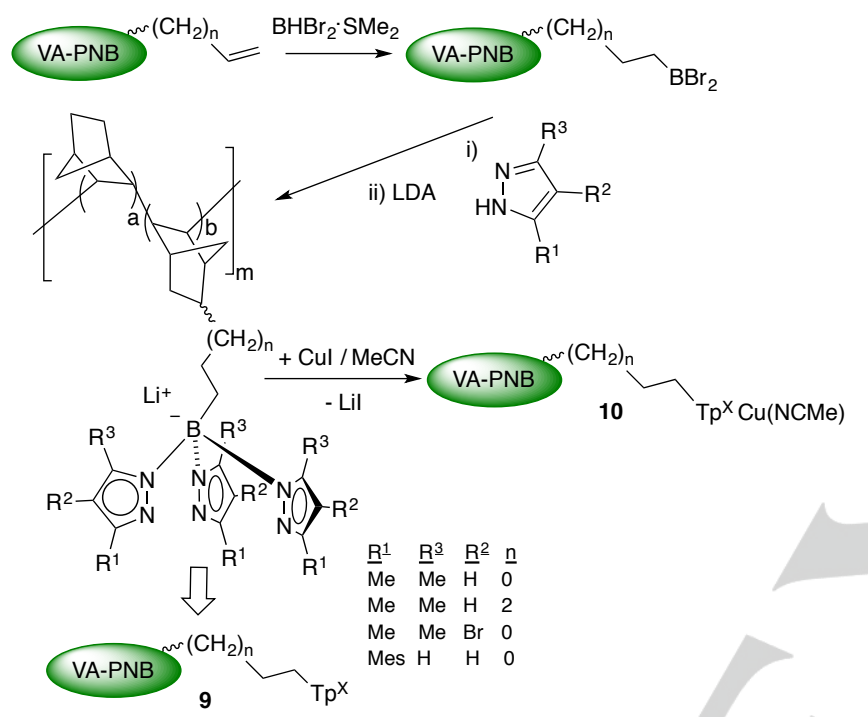

Scheme 13. Synthesis of VA-PNB-trispyrazolylborate ligands and their copper complexes.
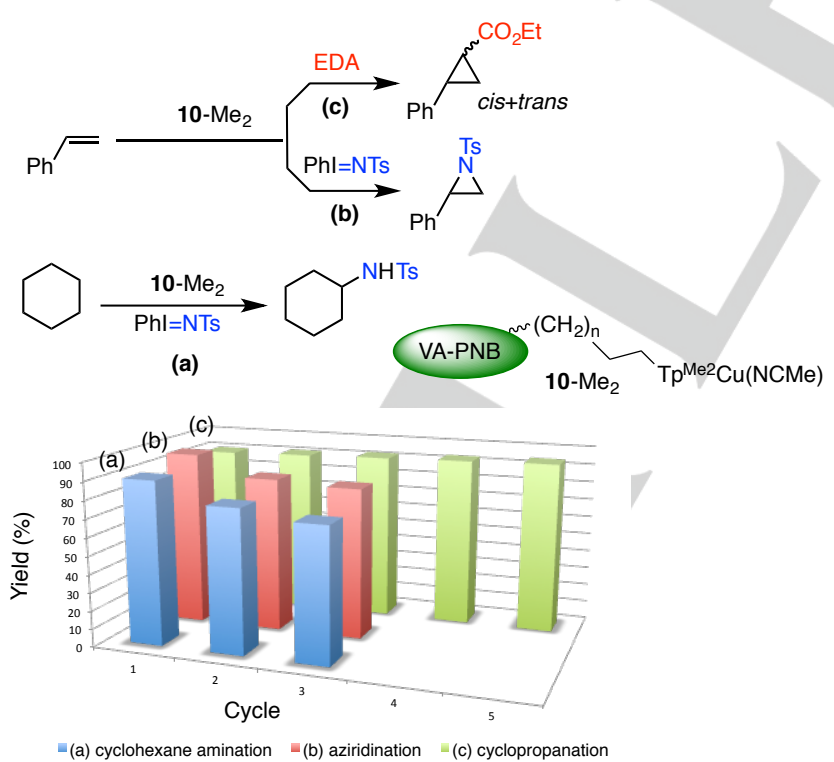

Scheme 14. Use of VA-PNB-supported trispyrazolilborate copper complexes in carbene and nitrene reactions.
The copper load in polymers 10 was very good, between 90$100 \%$ of the maximum load for most VA-PNB-Tp ${ }^{\mathrm{x}}$ s except the bulkiest 9-Mes $\left(\mathrm{R}^{1}=\right.$ mesityl) which was $68 \%$. The copper coordination environment in $\mathbf{1 0}$ was checked by IR spectroscopy of the analogous [VA-PNB-Tp ${ }^{x} \mathrm{Cu}(\mathrm{CO})$ ] complexes obtained by bubbling carbon monoxide through a suspension of 10 in dichloromethane. The $\mathrm{CO}$ absorption of these polymeric complexes showed the same wavenumber as the corresponding discrete complexes [ $\left.\mathrm{Tp}^{\mathrm{x}} \mathrm{Cu}(\mathrm{CO})\right]$. This shows that the Tp moiety anchored to VA-PNB is accessible for coordination to the metal and the suitable tricoordinated mode can be easily reached.

As shown in Scheme 14, complexes $\mathbf{1 0}$ are recyclable catalysts in cyclopropanation reactions and, albeit a small activity loss after the first cycle, also reusable for the aziridination of alkenes and the nitrene insertion into $\mathrm{C}-\mathrm{H}$ bonds. ${ }^{[22]}$

\section{Vinylic addition polynorbornenes as reagent support}

So far, the use of vinylic addition polynorbornenes as reagent support is circumscribed to the attachment of organotin compounds. Organostannanes are used as reagents in the palladium catalyzed Stille cross coupling reaction, in tinmediated radical processes as well as in allylation reactions and other applications. ${ }^{[30]}$ As a consequence of the toxicity of these compounds, that ranges from the more toxic methyl derivatives $\mathrm{SnMe}_{4-n} \mathrm{X}_{n}$ to the moderately harmful heavier alkyl substituted $\mathrm{SnBu}_{4-n} \mathrm{X}_{n},{ }^{[31]}$ the presence of even low levels of tin byproducts in synthetic products is unwanted. The tin contamination is a serious problem if the target product is a pharmaceutical derivative and, as a result, many of the useful synthetic protocols that use tin have become unpopular. However, an effective method of separation of the tin byproducts as well as their recovery and reuse, avoiding the generation of harmful waste, could solve the issue. This can be done by supporting the tin derivatives on a suitable polymer. ${ }^{[4 \mathrm{~d}, 30,32]}$

Most commonly used tin reagents are tributyl derivatives of the type $\mathrm{SnBu}_{3} \mathrm{R}(\mathrm{R}=$ hydrocarbyl group, $\mathrm{H}$ ). The $\mathrm{Sn}-\mathrm{Bu}$ bond is kinetically less reactive than other groups such as aryl, alkenyl, enolate or hydride. Therefore, a suitable support strategy would be to replace a butyl group on tin by a polymer that mimics it, i.e. an aliphatic scaffold such as VA-PNB.

Stannylated VA-PNBs have been synthesized following the routes depicted in Scheme 15. VA-PNB-SnBu $\mathrm{Cl}_{2}(\mathbf{1 1}$, Scheme $15)$ is the most versatile derivative that allows to synthesize a variety of vinylic addition polynorbornenes with $-\mathrm{SnBu}_{2} \mathrm{R}$ or $\mathrm{SnHBu}_{2}$ groups. Polymers 11 can be prepared by direct copolymerization reactions of norbornene and stannylated norbornenes catalyzed by $\left[\mathrm{Ni}\left(\mathrm{C}_{6} \mathrm{~F}_{5}\right)_{2}\left(\mathrm{SbPh}_{3}\right)_{2}\right]^{[24,33]}$ Also, the nucleophillic substitution reaction of a stannide derivative for bromide on the preformed VA-PNBs with pendant $\omega$-bromoalkyl groups is a very convenient route (Scheme 15). ${ }^{[24]}$ 


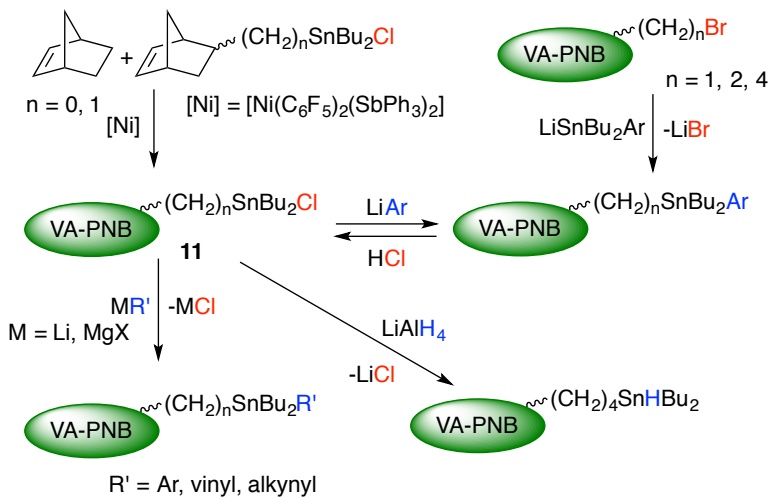

Scheme 15. Synthesis of stannylated VA-PNBs.

Different VA-PNB-SnBu ${ }_{2} \mathrm{R}$ polymeric reagents were used in the Stille reaction with good yields of the coupling products (Eq. $4),{ }^{[24,33]}$ and also in the tandem double Stille reaction shown in Eq. $5{ }^{[34]}$ In the latter case an improvement of the selectivity of the reaction towards the trans-allyl isomer shown was observed for the polymeric alkynyl tin reagents in comparison to the tributylalkynyltin derivatives. In all cases the use of VA-PNB$\mathrm{SnBu}_{2} \mathrm{R}$ reagents needed longer reaction times than those reported for the discrete $\mathrm{SnBu}_{3} \mathrm{R}$; this can be related to the lower solubility of the polymeric tin coupling partner and the accessibility of the tin centers. In fact, the longer the alkyl tether that separates the tin center from the polymeric backbone the higher the reactivity observed (trend in yields in Eq. 4: $n, 1<2<$ 4).
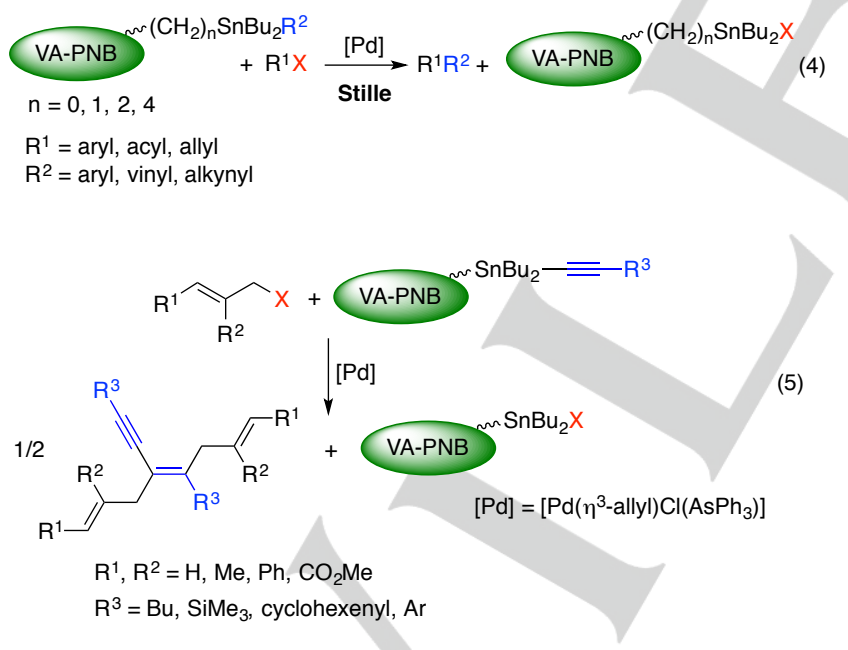

The VA-PNB-SnBu 2 X obtained as byproduct can be easily separated by filtration and it can be regenerated, by reaction with an organolithium derivative or a Grignard reagent, to be reused. The immobilization of the stannylated polymer in a packed column can be made and this is a convenient setup for batch operation multiple times (Scheme 16). After the Stille reaction of choice, the packed VA-PNB-SnBu ${ }_{2} \mathrm{X}$ polymer can be transformed by treatment with an organolithium derivative into
VA-PNB-SnBu ${ }_{2} \mathrm{R}$ and used again in the Stille reaction. Table 3 shows the results obtained for the coupling of allyl chloride and a polymeric aryl tin derivative. The most remarkable result in Table 3 is the very low tin contamination of the final products, as low as $15 \mathrm{ppm}$ (entries 2 and 6, Table 3). ${ }^{[2]}$ From this point just a few purification steps may afford the reaction product free from tin. This procedure avoids the usually cumbersome traditional separation methods of tin byproducts and the generation of waste. It stands in a very good position among all the strategies developed to tackle the problems connected to the management of tin reagents.

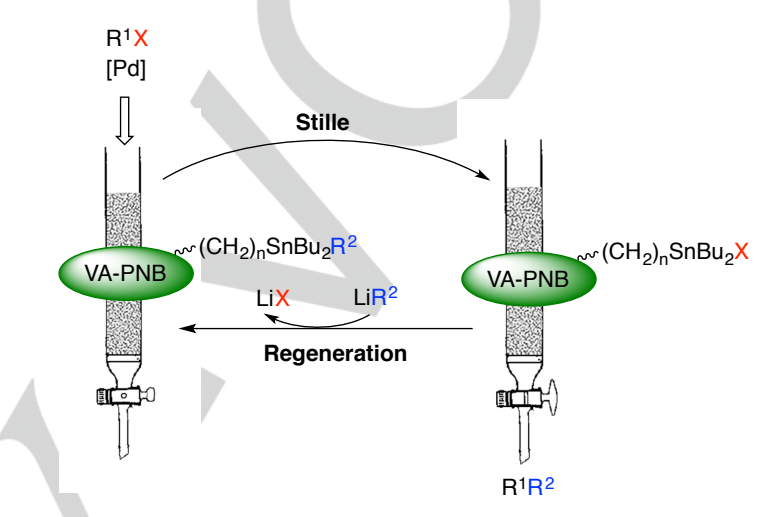

Scheme 16. Immobilized stannylated VA-PNBs as recyclable reagents in the Stille reaction.

Table 3. Reuse of VA-PNB-tin reagents in the Stille reaction using the setup shown in Scheme $16\left(n=4 ; R^{1}=\right.$ allyl; $\left.R^{2}=p-\mathrm{OMeC}_{6} \mathrm{H}_{4}\right)$.

\begin{tabular}{lll}
\hline Cycle & Isolated yield(\%) & Residual Sn (\% weight) \\
\hline 1 & 81 & 0.0029 \\
2 & 66 & 0.0015 \\
3 & 63 & 0.0045 \\
4 & 77 & 0.0026 \\
5 & 69 & 0.0034 \\
6 & 69 & 0.0015 \\
\hline
\end{tabular}

A more demanding application of supported stannane derivatives, as far as the polymer support is concerned, are radical reactions. In order to maintain the integrity of the polymeric scaffold, key to achieve recyclability, no sensitive positions to radical attack should be present. The aliphatic robust VA-PNBs are ideal for such a use and we explored the feasibility of developing a recyclable polymeric tin hydride.

A VA-PNB supported tin hydride is capable of effectively bringing about the stoichiometric dehalogenation reaction of 9bromophenanthrene and the resulting $\mathrm{VA}-\mathrm{PNB}-\mathrm{SnBu}_{2} \mathrm{Br}$ 
byproduct can be easily separated from the product by filtration (Eq. 6). ${ }^{[35]}$

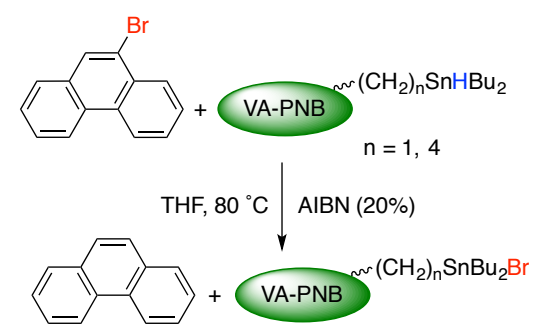

Given the low stability of tin hydrides, a more interesting approach is the use of a supported tin halide that can be converted in situ to the tin hydride by the action of a suitable hydride donor. This can be achieved using a mixture of polymethylhydrosiloxane (PMHS) and $\mathrm{KF}(\mathrm{aq})$, as depicted in Scheme 17, and using this approach the tin derivative can be used in substoichiometric amounts. ${ }^{[36]}$ The VA-PNB-SnBu $2 \mathrm{Cl}$ derivative 11 ( $n=4)$ was used for this purpose and it showed to be active in the dehalogenation reaction of a variety of aryl and alkyl bromides as well as in radical cyclization reactions.

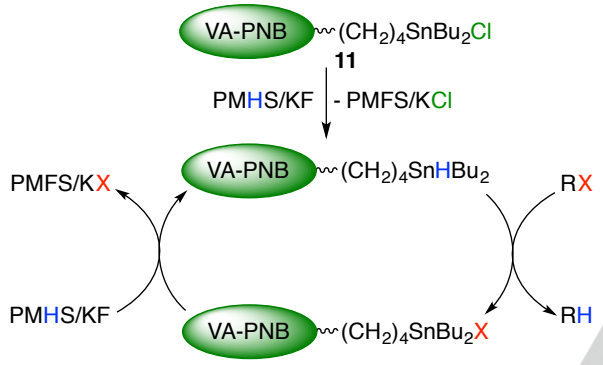

Scheme 17. In situ generation of the supported tin hydride using polymethylhydrosiloxane (PMHS) as hydride donor.

The polymer can be reused several times and, even if the yields obtained showed a noticeable fluctuation and a progressive efficiency loss, the stannylated VA-PNB remains active after many uses (Scheme 18), behaving as a stoichiometric reagent. The use of equimolar amounts of the supported reagent should not be a problem since the separation of the products is simple and the tin loss is very low. The analysis of the resulting polymer after several uses by solid state NMR showed the presence of $\mathrm{Sn}-\mathrm{F}$ and $\mathrm{Sn}-\mathrm{O}$ moieties that are active in subsequent reactions with the hydride donor. The presence of significant amounts of residual polysiloxanes in the polymer was also observed and this may be the main deactivation cause. It is worth noticing that the PMHS/KF mixture as hydride donor ensures that the reactions are tin-centered radical processes in contrast with borane derivatives, commonly used and intended to generate $\mathrm{Sn}-\mathrm{H}$ groups, which lead to boron-centered radicals and a different type of radical processes. The activity of many polymersupported tin reagents tested with boranes as hydride donors needs to be reevaluated. ${ }^{[32]}$ It is interesting to see the low residual tin amounts in the products, at least 150 times lower than those achieved using the conventional separation methods
(Scheme 18). The lower tin contamination as well as the easy separation procedure makes this protocol a useful one in tinmediated radical reactions, especially those that are involved in multistep syntheses with sensitive intermediates.
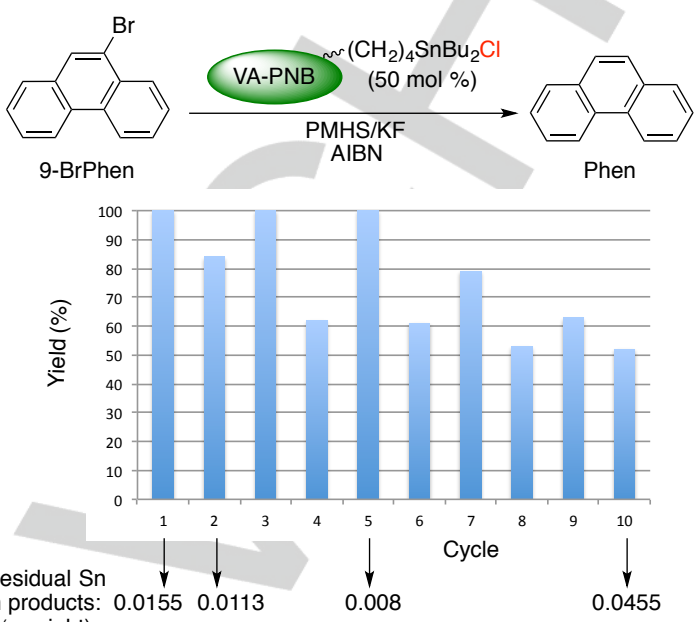

(\% weight)

Scheme 18. Multiple uses of VA-PNB-SnBu $2 \mathrm{X}$ in the radical dehalogenation of 9-bromophenathrene.

\section{Conclusions}

Vinylic addition polynorbornenes are robust scaffolds useful to support different types of catalysts and reagents in catalytic reactions. The thermal and chemical stability of this type or polymer makes the recyclability more feasible: the reuse of the supported material, either catalyst or byproduct, is not compromised by the chemical degradation of the polymer backbone or the loss of physical integrity of the support. The application of VA-PNBs in catalysis has not reached its full potential yet. However, there are enough examples in the literature that show that they are useful and they complement the commonly used organic polymers in more demanding applications (i.e. radical processes). The availability of a range of VA-PNB materials with functional groups amenable to postfunctionalization reactions makes the use of these polymers easy and should encourage the synthetic chemist, far from the polymer-chemistry area, to consider VA-PNBs as part of the toolbox in synthetic sustainable methodology.

\section{Acknowledgements}

We thank the financial support of the Spanish MINECO (SGPI, grant CTQ2016-80913-P) and the Junta de Castilla y León (grants VA062G18 and VA051P17 and a fellowship to RGL cofunded by the European Social Fund).

Keywords: polynorbornene $\cdot$ supported catalysis $\bullet$ cross coupling reactions $\cdot$ organocatalysts $\cdot$ metal catalysts 
[1] S. V. Ley, I. R. Baxendale, Nature Rev. 2002, 1, 573-586

[2] a) D. E. Bergbreiter, Chem. Rev. 2002, 102, 3345-3384; b) T. J. Dickerson, N. N. Reed, K. D. Janda, Chem. Rev. 2002, 102, 3325 3344 ; c) D. E. Bergbreiter, J. Tian, C. Hongfa, Chem. Rev. 2009, 109 530-582; d) Y. C. Yang, D. E. Bergbreiter, Pure Appl. Chem. 2013, 85 493-509.

[3] a) N. E. Leadbeater, M. Marco, Chem. Rev. 2002, 102, 3217-3274; b) C. A. McNamara, M. J. Dixon, M. Bradley, Chem. Rev. 2002, 102 3275-3300; c) S. Bräse, F. Lauterwasser, R. E. Ziegert, Adv. Synth Catal. 2003, 345, 869-929; d) B. M. L. Dioos, I. F. J. Vankelecom, P. A Jacobs, Adv. Synth. Catal. 2006, 348, 1413-1446; e) C. G. Frost, L. Mutton, Green Chem. 2010, 12, 1687-1703.

[4] a) Q. H. Fan, Y. M. Li, A. S. C. Chan, Chem. Rev. 2002, 102, 33853466; b) J. Lu, P. H. Toy, Chem. Rev. 2009, 109, 815-838; c) M. R. Buchmeiser, Chem. Rev. 2009, 109, 303-321; d) A. C. Albéniz, N Carrera, Eur. J. Inorg. Chem. 2011, 2347-2360; e) A. Molnàr, Chem. Rev. 2011, 111, 2251-2320.

[5] a) D. E. Bergbreiter, S. D. Sung, Adv. Synth. Catal. 2006, 348, 1352 1366; b) D. E. Bergbreiter, ACS Macro Lett. 2014, 3, 260-265.

[6] B. S. Lee, S. Mahajan, B. Clapham, K. D. Janda, J. Org. Chem. 2004, 69, 3319-3329.

[7] a) J. Pinaud, J. Vignolle, Y. Gnanou, D. Taton, Macromolecules 2011 , 44, 1900-1908; b) P. Coupillaud, J. Pinaud, N. Guidolin, J. Vignolle, M. Fèvre, E. Veaudecrenne, D. Mecerreyes, D. Taton, J. Polym. Sci. Part A: Polym. Chem. 2013, 51, 4530-4540.

[8] a) D. E. Bergbreiter, H. L. Su, H. Koizumi, J. Tian, J. Organomet. Chem. 2011, 696, 1272-1279; b) M. Al-Hashimi, C. Hongfa, B. George, H. S Bazzi, D. E. Bergbreiter, J. Polym. Sci. Part A: Polym. Chem. 2012, 50, 3954-3959.

[9] a) R. J. Colacot, W. A. Carole, B. A. Neide, A. Harad, Organometallics 2008, 27, 5605-5611.

[10] R. Madan, A. Srivastava, R. C. Anand, I. K. Varma, Prog. Polym. Sci. 1998, 23, 621-663.

[11] C. Janiak, P. G. Lassahn, J. Mol. Cat. A, 2001, 166, 193-209. F. Blank, C. Janiak, Coord. Chem. Rev. 2009, 253, 827-861. R. Ma, Y. Hou, J. Gao, F. Bao, J. Macromol. Science, part C: Pol. Rev. 2009, 49, 249287.

[12] a) A. D. Hennis, J. D. Polley, G. S. Long, A. Sen, D. Yandulov, J. Lipian, G. M. Benedikt, L. F. Rhodes, J. Huffman, Organometallics 2001, 20 , 2802-2812; b) D. A. Barnes, G. M. Benedikt, B. L. Goodall, S. S. Huang, H. A. Kalamarides, S. Lenhard, L. H. McIntosh, III, K. T. Selvy, R. A. Shick, L. F. Rhodes, Macromolecules 2003, 36, 2623-2632; c) M D. Walter, R. A. Moorhouse, S. A. Urbin, P. S. White, M. Brookhart, J. Am. Chem. Soc. 2009, 131, 9055-9069.

[13] C. W. Bielawski, R. H. Grubbs, Prog. Polym. Sci. 2007, 32, 1-29.

[14] a) W. J. Sommer, M. Weck, Adv. Synth. Catal. 2006, 348, 2101-2113; b) G. M. Pawar, M. R. Buchmeiser, Adv. Synth. Catal. 2010, 352, 917 928.

[15] S. J. Dolman, K. C. Hultzsch, F. Pezet, X. Teng, A. H. Hoveyda, R. R. Schrock, J. Am. Chem. Soc. 2004, 126, 10945-10953.

[16] R. García-Loma, A. C. Albéniz, RSC Adv. 2015, 5, 70244-70254.

[17] M. V. Bermeshev, P. P. Chapala, Prog. Polym. Sci. 2018, 84, 1-46.
[18] S. Martínez-Arranz, A. C. Albéniz, P. Espinet, Macromolecules 2010 43, 7482-7487.

[19] a) A. Bell, E. Elce, K. Seto, US Pat. 8765893 B2, 2014; b) B. Commarieu, J. P. Claverie, Chem. Sci. 2015, 6, 2172-2181; c) F. Pierre, B. Commarieu, A. C. Tavares, J. Claverie, Polymer 2016, 86, 91-97.

[20] S. Martínez-Arranz, E. Sánchez-Pérez, J. A. Molina de la Torre, Pérez-Ortega, A. C. Albéniz, RSC Adv. 2016, 6, 105878-105887.

[21] a) L. F. Rhodes, B. L. Goodall, R. Mülhaupt, A. Shick, G. M. Benedikt, S. K. Jayaraman, L. M. Soby, L. H. McIntosh, US Pat. 6294616B1, 2001; b) J. C. Ahn, S. H. Park, K. H. Lee, K. H. Park, Polym. Kor. 2003, 27, 429-435; c) H. Li, J. Li, Y. Zhang, Y. Mu, Polymer 2008, 49, 28392844; d) B. Commarieu, J. Potier, M. Compaore, S. Dessureault, B. L. Goodall, X. Li, J. P. Claverie, Macromolecules 2016, 49, 920-925.

[22] J. A. Molina de la Torre, I. Pérez-Ortega, A. Beltrán, M. R. Rodríguez, M. M. Díaz-Requejo, P. J. Pérez, A. C. Albéniz, Chem. Eur. J. 2018, 10.1002/chem.201803852.

[23] P. P. Chu, W. J. Huang, F. C. Chang S. Y. Fan, Polymer, 2000, 401404.

[24] S. Martínez-Arranz, N. Carrera, A. C. Albéniz, P. Espinet, A. VidalMoya, Adv. Synth. Catal. 2012, 354, 3551-3560.

[25] J. A. Molina de la Torre, A. C. Albéniz, ChemCatChem 2014, 6, 3547 3552.

[26] a) M. Rose, A. Notzon, M. Heitbaum, G. Nickerl, S. Paasch, E. Brunner F. Glorius, S. Kaskel, Chem. Commun. 2011, 47, 4814-4816; b) A. B. Powell, Y. Suzuki, M. Ueda, C. W. Bielawski, A. H. Cowley, J. Am. Chem. Soc. 2011, 133, 5218-5220; c) M. Tan, Y. Zhang, J. Y. Ying, Adv. Synth. Catal. 2009, 351, 1390-1394.

[27] I. K. Sagamanova, S. Sayalero, S. Martínez-Arranz, A. C. Albéniz, M. A. Pericàs, Catal. Sci. Technol. 2015, 5, 754-764.

[28] J. A. Molina de la Torre, A. C. Albéniz, ChemCatChem 2016, 8, 22412248.

[29] J. A. Molina de la Torre, A. C. Albéniz, Eur. J. Inorg. Chem. 2017 2911-2919.

[30] A. G. Davies, M. Gielen, K. H. Pannell, R. T. Tiekink (Eds.), Tin Chemistry: Fundamentals, Frontiers and Applications, Wiley, Chichester, 2008

[31] a) Y. Arakawa, O. Wada, T. H. Yu, Toxicol. Appl. Pharmacol. 1981, 60 , 1-7; b) I. J. Boyer, Toxicology, 1989, 55, 253-298; c) B. Buck, A. Mascioni, L. Que Jr., G. Veglia, J. Am. Chem. Soc. 2003, 125, 1331613317 and references therein; d) K. E. Apple, Drug Metab. Rev. 2004, 36, 763-786.

[32] For a comprehensive account of the separation methods of tin byproducts and the use of other modified or supported tin reagents see: E. Le Grognec, J.-M. Chrétien, F. Zammattio, J.-P. Quintard, Chem. Rev. 2015, 115, 10207-10260.

[33] N. Carrera, E. Gutiérrez, R. Benavente, M. M. Villavieja, A. C. Albéniz, P. Espinet, Chem. Eur. J. 2008, 14, 10141-10148.

[34] I. Meana, A. C. Albéniz, P. Espinet, Adv. Synth. Catal. 2010, 352, 2887 2891

[35] R. García-Loma, A. C. Albéniz, Eur. J. Org. Chem. 2017, 4247-4254.

[36] R. E. Maleczka, Jr. L. R. Terrell, D. H. Clark, S. L. Whitehead, W. P. Gallagher, I.Terstiege, J. Org. Chem. 1999, 64, 5958. 


\section{Entry for the Table of Contents}

\section{REVIEW}

A robust scaffold for sustainable catalysis can be found in vinylic addition polynorbornene (VA-PNB). Functionalized VA-PNBs with $\omega-$ bromoalkyl, bromoaryl or alkenyl pendant groups can be used to support recyclable organocatalysts, palladium or copper catalysts, as well as tin reagents in cross-coupling and radical reactions.

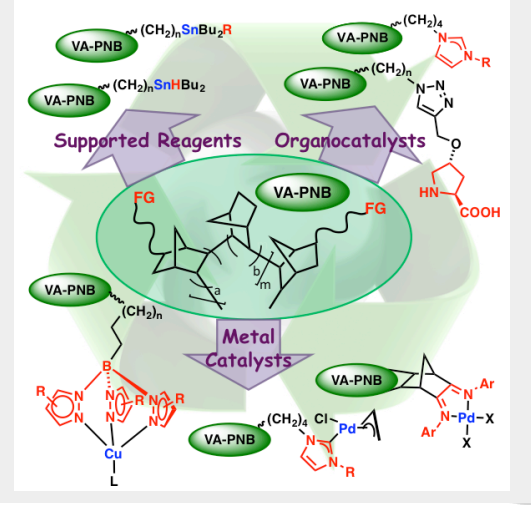

Rodrigo García-Loma and Ana C. Albéniz *l

Page No. - Page No.

Vinylic Addition Polynorbornene in Catalysis 